Pacific

Journal of

Mathematics

THE SIMULATION TECHNIQUE AND ITS APPLICATIONS TO INFINITARY COMBINATORICS UNDER THE AXIOM OF BLACKWELL DETERMINACY

BENEDIKT LöWE

Volume $214 \quad$ No. 2

April 2004 


\title{
THE SIMULATION TECHNIQUE AND ITS APPLICATIONS TO INFINITARY COMBINATORICS UNDER THE AXIOM OF BLACKWELL DETERMINACY
}

\author{
BENEDIKT LÖWE
}

\begin{abstract}
The Axiom of Blackwell Determinacy is a set-theoretic axiom motivated by games used in statistics. It is known that the Axiom of Determinacy implies the Axiom of Blackwell Determinacy. Tony Martin has conjectured that the two axioms are equivalent.

We develop the "simulation technique" which allows us to simulate boundedness proofs under the assumption of Blackwell Determinacy and deduce strong combinatorial consequences that can be seen as an important step towards proving Tony Martin's conjecture.
\end{abstract}

\section{Introduction.}

Set-theoretic game theory is an important part of Higher Set Theory. The research of the Cabal seminar and its successors unearthed deep connections between two-person perfect information games, inner models of set theory, and large cardinals.

The core concept of set-theoretic game theory is the notion of a strategy. Since set theorists usually worked with perfect information games, a strategy is a tree of moves in the set-theoretic setting. The strategy is a winning strategy for one of the two players if all its branches lie in the set designating a win for that player. Using the notion of a winning strategy, we can define when we call a set determined, viz. if one of the two players has a winning strategy.

For imperfect information games, this concept is too coarse. If one or both of the players are not completely informed about the current state of the game, we cannot expect winning strategies in the above sense to exist.

If we now look only at special classes of imperfect information (in this paper, this will be Blackwell games, or — as Blackwell calls them in [B197] — "Games with Slightly Imperfect Information"), we can define the notions of a mixed strategy and of strong optimality and use them to define the notion of Blackwell determinacy. Based on the notion of Blackwell determinacy, we can reformulate the questions of set-theoretic game theory for games of this type. 
The notion of Blackwell determinacy was introduced for finite games by von Neumann, and generalized to infinite games by David Blackwell [Bl69] who also proved the first theorems about Blackwell determinacy. At the MSRI Workshop in 1994 on Combinatorial Games, it was still open whether the Blackwell determinacy of all $G_{\delta \sigma}$ sets is a theorem of ZFC as Blackwell's extended abstract [B197] witnesses. Soon thereafter, Marco Vervoort proved the Blackwell determinacy of all $G_{\delta \sigma}$ sets in his Master's thesis [Ve95], and then Tony Martin developed a coding technique to derive Blackwell determinacy from perfect information determinacy in [Ma98]. In his Master's thesis, Vervoort also introduced the "Axiom of Blackwell Determinacy" which will be the protagonist of this paper.

In this paper, we shall discuss consequences of axioms of Blackwell Determinacy in the field of Infinitary Combinatorics. We shall define several axioms of Blackwell Determinacy in Section 2 and discuss Martin's conjecture on Blackwell Determinacy, Conjecture 2.6, which is the motivation for the rest of the paper. Section 2 is mostly expository and almost all results are either folklore or from the published literature.

In Section 3 we shall develop the simulation technique which will be used throughout this paper.

Section 4 is the main part of this paper, and its contents can be seen as an important step towards proving Conjecture 2.6. After introducing the basics of Infinitary Combinatorics under AD (Section 4.1), we transfer these results to Blackwell Determinacy using the simulation technique in Sections 4.2 and 4.3 . We prove that $\aleph_{1}$ has the strong partition property and that the odd projective ordinals are measurable cardinals. Some of the results of this paper have been announced together with additional results on a Blackwell Lipschitz hierarchy (without proof) in the survey paper [Lö02b].

The paper closes with a discussion of open problems in Section 5 .

\section{Definitions and notation.}

2.1. Set-theoretic standard notation. The notation used in this paper is standard. The reader is assumed to have a firm grasp of descriptive set theory and large cardinal theory as contained in [Mo80] and [Ka94]. All theorems and definitions that are not found in this paper can be found in one or both of the mentioned textbooks. Of course, we shall say reals or real numbers when we talk about elements of Baire space $\omega^{\omega}$ as is customary in set theory.

Since Blackwell determinacy contradicts the full Axiom of Choice AC, we shall work throughout this paper in the theory

$$
Z F+D C \text {. }
$$


We shall need DC in the context of Infinitary Combinatorics, yet for most of this paper, $A C_{\omega}(\mathbb{R})$ will be enough. Note that its an open problem whether $\mathrm{AC}_{\omega}(\mathbb{R})$ follows from Blackwell determinacy (as it does from $A D$ ).

We now fix some notation. In the following, let $X \subseteq \omega$ be the set of possible moves.

Let us write $X^{\text {even }}:=\left\{s \in X^{<\omega} ; \operatorname{lh}(s)\right.$ is even $\}, X^{\text {odd }}:=\left\{s \in X^{<\omega} ; \operatorname{lh}(s)\right.$ is odd $\}$, and $\operatorname{Prob}(X)$ for the set of probability measures on $X$.

Fix a recursive bijection $\ulcorner\cdot, \cdot\urcorner: \omega \times \omega \rightarrow \omega$. If $x \in X^{\omega}$, we define $(x)_{i}(n):=$ $x(\ulcorner i, n\urcorner)$. Using this notation we can easily code countable sequences of elements of $X^{\omega}$ into one element of $X^{\omega}$.

We shall be using the standard notation for infinite games: If $x \in X^{\omega}$ is the sequence of moves for player I and $y \in X^{\omega}$ is the sequence of moves for player II, we let $x * y$ be the sequence constructed by playing $x$ against $y$, i.e.,

$$
(x * y)(n):= \begin{cases}x(k) & \text { if } n=2 k \\ y(k) & \text { if } n=2 k+1 .\end{cases}
$$

Conversely, if $x \in X^{\omega}$ is a run of a game, then we let $x_{\mathrm{I}}$ be the part played by player I and $x_{\mathrm{II}}$ be the part played by player II, i.e., $x_{\mathrm{I}}(n)=x(2 n)$ and $x_{\mathrm{II}}(n)=x(2 n+1)$. We shall extend this notation to sets $A \subseteq X^{\omega}$ in the obvious way: $A_{\mathrm{I}}:=\left\{x_{\mathrm{I}} ; x \in A\right\}$ and $A_{\mathrm{II}}:=\left\{x_{\mathrm{II}} ; x \in A\right\}$. If $Y \subseteq X^{\omega}$, we write $A_{Y}^{\mathrm{I}}$ for the set $\left\{x \in A ; x_{\mathrm{II}} \in Y\right\}$ and $A_{Y}^{\mathrm{II}}$ for the set $\left\{x \in A ; x_{\mathrm{I}} \in Y\right\}$. (In most situations, $Y$ will be of the form $\{y\}$.) We denote by WO the $\Pi_{1}^{1}$-complete set of all codes of wellorderings relative to the bijection $\ulcorner\cdot, \cdot\urcorner$.

A standard result that we shall be using a lot is the Boundedness Lemma in the following abstract form:

Theorem 2.1. Let $\boldsymbol{\Gamma} \subseteq \wp\left(X^{\omega}\right)$ be a pointclass with the following properties:

- $\boldsymbol{\Gamma}$ is boldface (i.e., closed under continuous preimages),

- $\boldsymbol{\Gamma}$ has the prewellordering property, and

- $\boldsymbol{\Gamma}$ is closed under $\forall^{\mathbb{R}}$ and finite unions.

Suppose that $X$ is a $\boldsymbol{\Gamma}$-complete set, $\varphi$ is a $\boldsymbol{\Gamma}$ prewellordering on $X$, and $A \subseteq X$ is $\breve{\boldsymbol{\Gamma}}$.

Then $A$ is bounded in the following sense: There is an $\alpha<\operatorname{lh}(\varphi)$ such that for all $a \in A, \varphi(a)<\alpha$.

Proof. First of all, note that the assumptions on $\boldsymbol{\Gamma}$ imply that $\boldsymbol{\Gamma}$ is nonselfdual (no selfdual pointclass can have the prewellordering property, [Ka94, Exercise 29.2, Proposition 29.3 \& Proposition 29.7]). The closure properties of $\boldsymbol{\Gamma}$ give that $\breve{\boldsymbol{\Gamma}}$ is boldface, closed under $\exists^{\mathbb{R}}$ and finite intersections.

Now we assume that boundedness fails and derive a contradiction: Let $A \in \breve{\boldsymbol{\Gamma}}$ be such that for all $\alpha<\operatorname{lh}(\varphi)$ there is $a \in A$ with $\varphi(a)>\alpha$. Then (as in the standard proof of boundedness), we can show that every set in $\boldsymbol{\Gamma}$ is in $\breve{\boldsymbol{\Gamma}}$ thereby showing that $\boldsymbol{\Gamma}$ is selfdual, which is a contradiction. 
2.2. Blackwell games. As mentioned in the Introduction, we shall not be dealing with imperfect information games in full generality, but with the very special subclass that Blackwell called "Games with Slightly Imperfect Information", and we shall call Blackwell games. We imagine a perfect information game in which both players have to move simultaneously in each step. Consequently, player II has only partial information: He is aware of all the game information up to the current step, but doesn't know what player I plays in the current round. This situation is modelled by the notion of a Blackwell strategy (see below).

We call a function $\sigma: X^{\text {Even }} \rightarrow \operatorname{Prob}(X)$ a mixed strategy for player I and a function $\sigma: X^{\text {Odd }} \rightarrow \operatorname{Prob}(X)$ a mixed strategy for player II.

Let us describe two particularly interesting types of mixed strategies:

- A mixed strategy is called Blackwell strategy if it doesn't depend on the moves in the same turn, i.e., if $s$ and $t$ have the same longest even subsequence, then we have $\sigma(s)=\sigma(t)$.

- A mixed strategy $\sigma$ is called pure if for all $s \in \operatorname{dom}(\sigma)$ the measure $\sigma(s)$ is a Dirac measure, i.e., there is a natural number $n$ such that $\sigma(s)(\{n\})=1$. (This is of course equivalent to being a strategy in the perfect information sense.)

Let

$$
\nu(\sigma, \tau)(s):= \begin{cases}\sigma(s) & \text { if } \operatorname{lh}(s) \text { is even, and } \\ \tau(s) & \text { if } \operatorname{lh}(s) \text { is odd. }\end{cases}
$$

Then for any $s \in \omega^{<\omega}$, we can define

$$
\mu_{\sigma, \tau}([s]):=\prod_{i=0}^{\ln (s)-1} \nu(\sigma, \tau)\left(s\lceil i)\left(\left\{s_{i}\right\}\right) .\right.
$$

This generates a Borel probability measure on $\omega^{\omega}$ which can be seen as an indicator of how well the strategies $\sigma$ and $\tau$ perform against each other. If $B$ is a Borel set, $\mu_{\sigma, \tau}(B)$ is interpreted as the probability that the result of the game ends up in the set $B$ when player I randomizes according to $\sigma$ and player II according to $\tau$. Note that if $\sigma$ and $\tau$ are both pure, then $\mu_{\sigma, \tau}$ is a Dirac measure concentrated on the unique real that is the outcome of this game, denoted by $\sigma * \tau$.

If $\sigma$ and $\tau$ are Blackwell strategies, the game modelled by $\sigma$ and $\tau$ corresponds to the "Games with Slightly Imperfect Information" of [B197]: Both players can be understood as moving simultaneously, so player II may not use any information about the move of player $\mathrm{I}$ in the same round of the game.

- We call a pure strategy $\sigma$ for player I ( $\tau$ for player II) a winning strategy if for all pure counterstrategies $\tau(\sigma)$, we have that $\sigma * \tau \in A$ $(\sigma * \tau \notin A)$. 
- We define a measure of quality (the mixed value of the strategy) for mixed strategies $\sigma$ (for player I) or $\tau$ (for player II) by $\operatorname{mval}_{\mathrm{I}}^{A}(\sigma):=\inf \left\{\mu_{\sigma, \tau}^{-}(A) ; \tau\right.$ is a mixed strategy for player II $\}$, and $\operatorname{mval}_{\mathrm{II}}^{A}(\tau):=\sup \left\{\mu_{\sigma, \tau}^{+}(A) ; \sigma\right.$ is a mixed strategy for player I $\} .{ }^{1}$

- Also for Blackwell strategies $\sigma$ (for player I) or $\tau$ (for player II), we define a measure of quality (the Blackwell value of the strategy) by

$\operatorname{Bval}_{\mathrm{I}}^{A}(\sigma):=\inf \left\{\mu_{\sigma, \tau}^{-}(A) ; \tau\right.$ is a Blackwell strategy for player II $\}$, and

$\operatorname{Bval}_{\mathrm{II}}^{A}(\tau):=\sup \left\{\mu_{\sigma, \tau}^{+}(A) ; \sigma\right.$ is a Blackwell strategy for player I $\}$.

We call a set $A$ determined if either player I or player II has a winning strategy, and we call a pointclass $\Gamma$ determined if all sets in $\Gamma$ are determined (in symbols: $\operatorname{Det}(\Gamma)$ ).

We define the mixed and Blackwell value sets for player I and player II by

$V_{\mathrm{I}}^{\operatorname{mix}}(A):=\left\{\operatorname{mval}_{\mathrm{I}}^{A}(\sigma) ; \sigma\right.$ is a mixed strategy for player $\left.\mathrm{I}\right\}$, and

$V_{\mathrm{II}}^{\operatorname{mix}}(A):=\left\{\operatorname{mval}_{\mathrm{II}}^{A}(\tau) ; \tau\right.$ is a mixed strategy for player II $\}$,

$V_{\mathrm{I}}^{\mathrm{Bl}}(A):=\left\{\operatorname{Bval}_{\mathrm{I}}^{A}(\sigma) ; \sigma\right.$ is a Blackwell strategy for player $\left.\mathrm{I}\right\}$, and

$V_{\mathrm{II}}^{\mathrm{Bl}}(A):=\left\{\mathrm{Bval}_{\mathrm{II}}^{A}(\tau) ; \tau\right.$ is a Blackwell strategy for player II $\}$.

Then $V_{\mathrm{II}}^{\operatorname{mix}}(A)$ lies entirely above $V_{\mathrm{I}}^{\operatorname{mix}}(A)$ in the sense that for all $v \in$ $V_{\mathrm{II}}^{\operatorname{mix}}(A)$ and $v^{*} \in V_{\mathrm{I}}^{\operatorname{mix}}(A)$ we have $v \geq v^{*}$ (and the same for $V_{\mathrm{I}}^{\mathrm{Bl}}(A)$ and $\left.V_{\mathrm{II}}^{\mathrm{Bl}}(A)\right)$.

If now $\inf V_{\mathrm{II}}^{\mathrm{Bl}}(A)=\sup V_{\mathrm{I}}^{\mathrm{Bl}}(A)=: p$, then the outcome of the game is stochastically determined as follows: Both players can approximate the outcome that player I wins with probability $p$. In this case, we call the payoff set (imperfect information) Blackwell determined. Similarly, if inf $V_{\mathrm{II}}^{\operatorname{mix}}(A)=\sup V_{\mathrm{I}}^{\operatorname{mix}}(A)$, we call the payoff set $A$ perfect information Blackwell determined.

It is well-known that for infinite sets $X$ it's easy to construct clopen payoff sets $A \subseteq X^{\omega}$ such that $A$ is not imperfect information Blackwell determined. Thus, in order to talk about this property, we have to restrict ourselves to looking at $A \subseteq X^{\omega}$ where $X$ is finite. For a pointclass $\Gamma$, we say it's (imperfect information) Blackwell determined (in symbols: BI-Det $(\Gamma)$ ) if all sets $A \in \Gamma$ with $A \subseteq X^{\omega}$ are (imperfect information) Blackwell determined (where $X$ is a finite set), and we say that it is perfect information Blackwell determined (in symbols: pBI-Det $(\Gamma)$ ) if for all $A \in \Gamma$, the set $A$ is perfect information Blackwell determined.

\footnotetext{
${ }^{1}$ Here, $\mu^{+}$denotes outer measure and $\mu^{-}$denotes inner measure with respect to $\mu$ in the usual sense of measure theory. If $A$ is Borel, then $\mu^{+}(A)=\mu^{-}(A)=\mu(A)$ for Borel measures $\mu$.
} 
In 2000, Martin and Vervoort proved a crucial result about games with mixed strategies [MaNeVe03, Lemma 3.7]:

Theorem 2.2 (Martin-Vervoort Zero-One Law, 2000). If $A \subseteq X^{\omega}$ is perfect information Blackwell determined. Then $\inf V_{\mathrm{II}}^{\operatorname{mix}}(A)=\sup V_{\mathrm{I}}^{\operatorname{mix}}(A)$ is either 0 or 1.

A mixed strategy for player I is now called strongly optimal for $A$ if $\operatorname{mval}_{\mathrm{I}}^{A}(\sigma)=1$, and and a mixed strategy $\tau$ for player II is called strongly optimal for $A$ if $\operatorname{mval}_{\mathrm{II}}^{A}(\tau)=0$.

Vervoort was able to use the Martin-Vervoort Zero-One-Law to prove the existence of strongly optimal strategies [MaNeVe03, Lemma 3.10] (cf. also [Lö02a] for a transfer of a finite branching version of Theorem 2.3 to an infinite branching version):

Theorem 2.3. Let $\boldsymbol{\Gamma}$ be a boldface pointclass. Suppose that $\mathrm{pBI}-\operatorname{Det}(\boldsymbol{\Gamma})$ holds and $A \in \boldsymbol{\Gamma}$ where $A$ is a subset of $\omega^{\omega}$. Then there is either a strongly optimal strategy for player I or a strongly optimal strategy for player II in the game with payoff $A$.

Corollary 2.4. Let $\boldsymbol{\Gamma}$ be a boldface pointclass. Then $\mathrm{pBI}-\operatorname{Det}(\boldsymbol{\Gamma})$ is equivalent to "for each $A \in \Gamma$, either player I or player II has a strongly optimal strategy."

We now defined three notions of determinacy allowing us to look at three different axioms of determinacy $\mathrm{AD}, \mathrm{BI}-\mathrm{AD}$, and $\mathrm{pBI}-\mathrm{AD}$ (and their restrictions to the projective sets PD, BI-PD and pBI-PD):

- The Axiom of Determinacy AD: All subsets of $\omega^{\omega}$ are determined.

- The Axiom of (imperfect information) Blackwell Determinacy BI-AD: All subsets of $2^{\omega}$ are (imperfect information) Blackwell determined.

- The Axiom of perfect information Blackwell Determinacy pBI-AD: All subsets of $\omega^{\omega}$ are perfect information Blackwell determined.

To these three axioms, we shall add another one: The Axiom of blindfolded Blackwell Determinacy. Instead of defining optimality by looking at all mixed counterstrategies, we can look at smaller classes of counterstrategies. One class that will happen to play a rôle is the class of all trivial strategies:

We call a mixed strategy $\sigma$ trivial, if it is a pure strategy and it's values don't depend on the input, i.e., whenever $s$ and $t$ are sequences of the same length, then $\sigma(s)=\sigma(t)$. A trivial strategy corresponds to a real that is fixed in advance before a single move of the game is played and which the player following the strategy is using as his predetermined moves. Playing according to a trivial strategy is like playing blindfoldedly: You have no idea what is going on in the game and just follow a previously fixed sequence of moves no matter what happens. 
Now we call a mixed strategy $\sigma$ for player I (player II) weakly optimal if for all trivial counterstrategies $\tau$, we have $\mu_{\sigma, \tau}^{-}(A)=1\left(\mu_{\tau, \sigma}^{+}(A)=0\right)$. The existence of weakly optimal strategies is a very weak property. It is possible that both players have weakly optimal strategies, and it's even possible that player I has a weakly optimal strategy, but player II has a winning strategy.

We say a set $A$ is blindfoldedly Blackwell determined if one of the two players has a weakly optimal strategy. A pointclass $\Gamma$ is called blindfoldedly Blackwell determined if all sets $A \in \Gamma$ are blindfoldedly Blackwell determined (in symbols: bIBI-Det $(\Gamma)$ ). Finally, the Axiom of blindfolded Blackwell determinacy bIBI-AD says that all sets are blindfoldedly Blackwell determined. To make this axiom sound less bizarre, let us note that for a pure strategy $\sigma$, being winning and being winning against all trivial counterstrategies are equivalent, so that a notion of "blindfolded determinacy" would be equivalent to determinacy in the usual perfect information setting in a very strong sense.

We now have four determinacy axioms - what are the relations between them?

\section{Theorem 2.5.}

(a) AD implies $\mathrm{BI}-\mathrm{AD}$,

(b) $\mathrm{BI}-\mathrm{AD}$ is equivalent to $\mathrm{pBI}-\mathrm{AD}$, and

(c) $\mathrm{pBI}-\mathrm{AD}$ implies bIBI-AD.

Proof. (a) is an instance of the main theorem of [Ma98] and (c) is obvious.

For the " $\Rightarrow$ "-direction of (b), we model a game with mixed strategies by a game with Blackwell strategies in which the players pass every second move. The " $\Leftarrow$ "-direction follows from the remarks on [Ma98, p. 1579] and [MaNeVe03, p. 618sq.]: The main theorem of [Ma98] can be proved using mixed strategies.

The main open question in this area which is also the motivation for the work in this paper is Tony Martin's conjecture that the converse of Theorem 2.5 (a) also holds:

Conjecture 2.6. BI-AD implies AD.

Tony Martin, Itay Neeman and Marco Vervoort proved (cf. [MaNeVe03, Theorems $5.1 \& 5.7]$ ) the following result, thereby determining the consistency strength of pBI-AD:

Theorem 2.7 (Martin-Neeman-Vervoort 1999/2000). pBI-PD implies PD and $(\mathrm{pBI}-\mathrm{AD})^{\mathbf{L}(\mathbb{R})}$ implies $(\mathrm{AD})^{\mathbf{L}(\mathbb{R})}$.

However, while their result yields the equiconsistency of $\mathrm{pBI}-\mathrm{AD}$ and $\mathrm{AD}$, it doesn't give an equivalence. So far, Conjecture 2.6 is open. In this paper we shall get a rich structure theory of the ordinals up to $\aleph_{\omega+1}$ that is usually 
seen as being very characteristic of $A D$ and thus can serve as an indication that Martin's Conjecture 2.6 is true.

Let us close by mentioning that the usual proof of Lebesgue measurability from determinacy doesn't work with Blackwell determinacy. Vervoort proved (using a new proof, cf. [Ve95, Theorem 6.11]) that BI-Det( $\boldsymbol{\Gamma})$ implies that every set in $\boldsymbol{\Gamma}$ is Lebesgue measurable. Thus we have (by standard arguments):

Theorem 2.8. Assume BI-AD and let $\mu$ be a $\sigma$-finite measure (in particular, all of our measures $\mu_{\sigma, \tau}$ derived from mixed strategies $\sigma$ and $\tau$ will do). Let $\left\langle B_{\xi} ; \xi<\lambda\right\rangle$ be a wellordered sequence of sets with $\mu\left(B_{\xi}\right)=1$. Then $\mu\left(\bigcap_{\xi<\lambda} B_{\xi}\right)=1$.

Let us sum up the implications between our axioms of determinacy and some other set-theoretic statements as they were known before this paper in Figure 1. Dotted lines indicate results proved in this paper; $\mathbf{M}^{\text {strong }}$ abbreviates "there is an inner model with a strong cardinal".

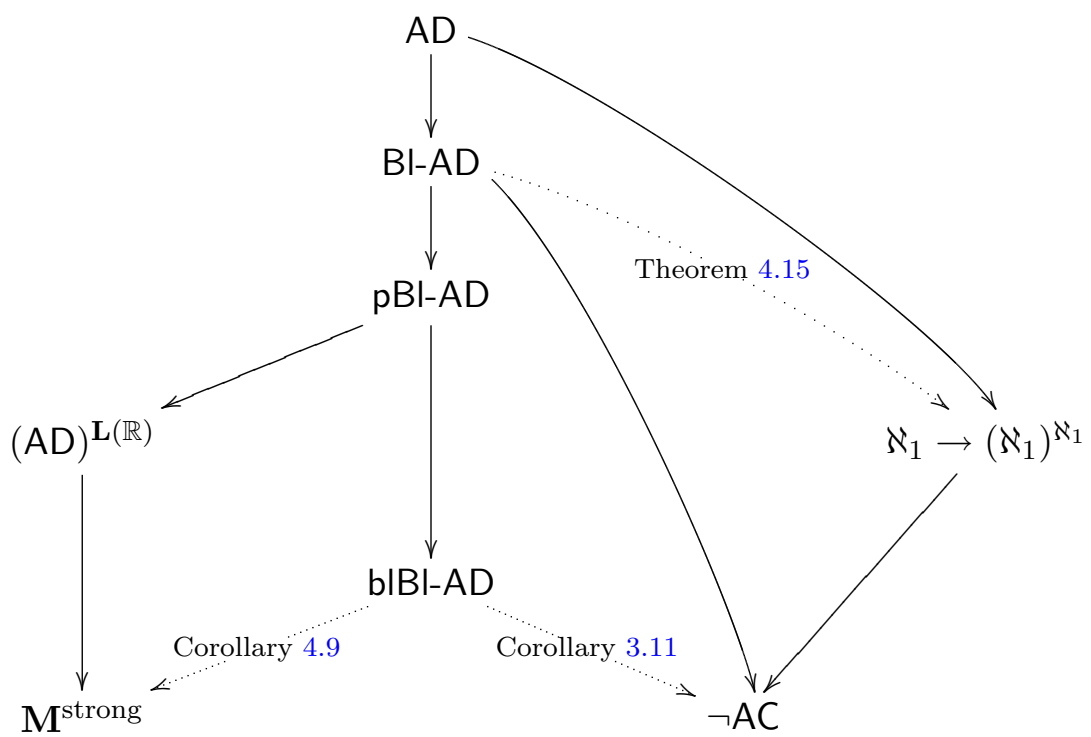

Figure 1. Diagram of axioms of determinacy and consequences.

\section{The simulation technique.}

In this section we shall introduce a technique called the simulation technique. This technique will be our main tool in getting consequences from Blackwell determinacy. 
Many proofs of some statement AD $\rightarrow \Psi$ involve some game $G$ and split up into two cases: Either player I has a winning strategy in the game $G$ (we denote this situation by $\Phi_{\mathrm{I}}$ ) or player II has a winning strategy in the game $G$ (we denote this situation by $\Phi_{\mathrm{II}}$ ). The proof then proceeds by showing $\Phi_{\mathrm{I}} \rightarrow \Psi_{\mathrm{I}}$ and $\Phi_{\mathrm{II}} \rightarrow \Psi_{\mathrm{II}}$, where $\Psi_{\mathrm{I}} \vee \Psi_{\mathrm{II}}$ implies $\Psi$ (very often, $\Psi_{\mathrm{I}} \equiv 0=1$ and $\left.\Psi_{\mathrm{II}} \equiv \Psi\right)$.

Now, if we want to simulate these proofs to get a proof of $\mathrm{pBI}-\mathrm{AD} \rightarrow \Psi$, we weaken the two cases to $\Phi_{\mathrm{I}}^{*}$ ("Player I has a strongly optimal strategy") and $\Phi_{\mathrm{II}}^{*}$ ("Player II has a strongly optimal strategy"), and need to show that $\Phi_{\mathrm{I}}^{*} \rightarrow \Psi_{\mathrm{I}}$ and $\Phi_{\mathrm{II}}^{*} \rightarrow \Psi_{\mathrm{II}}$ are still provable.

The simulation technique allows to do this in special cases. In particular, the simulation technique does not generate essentially new proofs but proves that in some situations, the classical AD proofs can be simulated in the Blackwell context.

\subsection{Kechris-Tanaka pointclasses \& pseudoimages.}

Definition 3.1. Let $\boldsymbol{\Gamma}$ be a boldface pointclass. We shall call it a KechrisTanaka pointclass if:

(KT1) $\boldsymbol{\Gamma}$ is closed under existential real quantification,

(KT2) for all $A \subseteq\left(\omega^{\omega}\right)^{2}$ with $A \in \boldsymbol{\Gamma}$, and all reals $\varepsilon$ the sets

$$
\begin{gathered}
\left\{\langle x, \sigma, \tau\rangle ; \mu_{\sigma, \tau}^{-}\left(A_{x}\right)>\varepsilon\right\} \text { and } \\
\left\{\langle x, \sigma, \tau\rangle ; \mu_{\sigma, \tau}^{+}\left(A_{x}\right)>\varepsilon\right\}
\end{gathered}
$$

are in $\boldsymbol{\Gamma}$, and

(KT3) $\quad \boldsymbol{\Gamma}$ is closed under countable intersections.

We say that a boldface pointclass $\boldsymbol{\Gamma}$ has the weak scale property if every set in $\boldsymbol{\Gamma}$ admits a $\forall^{\mathbb{R}} \breve{\boldsymbol{\Gamma}}$-scale (this should not be confused with the weak scale property of [MaNeVe03]). Under PD, every universal projective class has the weak scale property, and that the class of Borel sets has the weak scale property (without any assumptions).

Theorem 3.2 (Kechris-Tanaka). Suppose $\boldsymbol{\Gamma}$ is a boldface pointclass closed under countable intersections. Suppose that there is a pointclass $\boldsymbol{\Gamma}^{*}$ with the following properties:

1) $\exists{ }^{\mathbb{R}} \boldsymbol{\Gamma}^{*}=\boldsymbol{\Gamma}$,

2) $\Gamma^{*}$ has the weak scale property, and

3) every set in $\boldsymbol{\Gamma}^{*}$ is Lebesgue measurable.

Then $\boldsymbol{\Gamma}$ is a Kechris-Tanaka pointclass.

A proof can be found in [Ke73, Theorem 2.2.3 \& Corollary 2.2.2]. Theorem 3.2 yields that (under the assumption of PD for $n>1$ ) $\boldsymbol{\Sigma}_{n}^{1}$ is a KechrisTanaka pointclass. 
Let $A \subseteq \omega^{\omega}$ be a set of reals and $\leq$ any prewellordering of $\omega^{\omega}$. Also fix a mixed strategy $\sigma$ for player I and a mixed strategy $\tau$ for player II.

Let $U_{x}^{\leq, \mathrm{I}}:=\left\{u ; x_{\mathrm{I}} \leq u_{\mathrm{I}}\right\}$ and $U_{x}^{\leq, \mathrm{II}}:=\left\{u ; x_{\mathrm{II}} \leq u_{\mathrm{II}}\right\}$. Using this notation, we define the $\leq$-pseudoimage of $A$ under $\sigma$ (under $\tau$ ):

$$
\begin{gathered}
\Psi_{\leq}^{\sigma, \mathrm{I}}(A):=\left\{x_{\mathrm{I}} ; \exists z \in A\left(\mu_{\sigma, z}^{-}\left(U_{x}^{\leq, \mathrm{I}}\right)>0\right)\right\}, \text { and } \\
\Psi_{\leq}^{\tau, \mathrm{II}}(A):=\left\{x_{\mathrm{II}} ; \exists z \in A\left(\mu_{z, \tau}^{+}\left(U_{x}^{\leq, \mathrm{II}}\right)>0\right)\right\} .
\end{gathered}
$$

Proposition 3.3. If $\boldsymbol{\Gamma}$ is a Kechris-Tanaka pointclass, and $\leq$ and $A$ are in $\boldsymbol{\Gamma}$, then for all strategies $\sigma$ for player $I$ and $\tau$ for player $I I$, both $\Psi_{\leq}^{\sigma, \mathrm{I}}(A)$ and $\Psi_{\leq}^{\tau, \mathrm{II}}(A)$ are in $\boldsymbol{\Gamma}$.

Proof. The sets $U_{x}^{\leq, \mathrm{I}}$ and $U_{x}^{\leq, \mathrm{II}}$ are in $\boldsymbol{\Gamma}$, so (KT1) and (KT2) give us that the pseudoimage is in $\exists \mathbb{R} \boldsymbol{\Gamma}=\boldsymbol{\Gamma}$.

Proposition 3.4. Let $\leq$ be a prewellordering of $\omega^{\omega}$, and $Y$ an arbitrary nonempty set of reals. If $\sigma$ is a strongly optimal strategy for player I ( $\tau$ a strongly optimal strategy for player II) for the set A, then

$$
\Psi_{\leq}^{\sigma, \mathrm{I}}(Y) \cap\left(A_{Y}^{\mathrm{I}}\right)_{\mathrm{I}} \neq \emptyset \quad\left(\Psi_{\leq}^{\tau, \mathrm{II}}(Y) \cap\left(A_{Y}^{\mathrm{II}}\right)_{\mathrm{II}} \neq \emptyset\right) .
$$

Proof. Let $y \in Y$. Since $\sigma$ is strongly optimal, we know that $\mu_{\sigma, y}^{-}(A)=$ $\mu_{\sigma, y}^{-}\left(A_{Y}^{\mathrm{I}}\right)=1$. Let now $x \in A_{Y}^{\mathrm{I}}$ such that the $\leq$-rank of $x_{\mathrm{I}}$ is minimal. Clearly, $\mu_{\sigma, y}^{-}\left(U_{x}^{\leq, \mathrm{I}}\right)=1$, and so $x_{\mathrm{I}} \in \Psi_{\leq}^{\sigma, \mathrm{I}}(Y)$.

With the notion of pseudoimage at hand, we can prove a rather abstract version of the boundedness lemma for the following type of games:

Definition 3.5. Let $A \subseteq \omega^{\omega}$. Then $A$ is called of boundedness type if there is a boldface Kechris-Tanaka pointclass $\boldsymbol{\Gamma}$ such that $\breve{\boldsymbol{\Gamma}}$ has the prewellordering property and a set $X \in \breve{\boldsymbol{\Gamma}} \backslash \boldsymbol{\Gamma}$ with $\breve{\boldsymbol{\Gamma}}$-norm $\varphi: X \rightarrow \alpha$ such that:

(B1) $\quad x_{\mathrm{I}} \notin X$ implies $x \notin A$, and

(B2) there is a cofinal function $\varrho: \omega^{\omega} \rightarrow \alpha$ with the property that $\varrho\left(x_{\mathrm{II}}\right) \geq \varphi\left(x_{\mathrm{I}}\right)$ implies $x \notin A$.

Theorem 3.6 (Boundedness Lemma). Assume pBI-AD. Let $A$ be of boundedness type. Then there is a strongly optimal strategy for player II.

Proof. Let $\boldsymbol{\Gamma}, X \in \breve{\boldsymbol{\Gamma}} \backslash \boldsymbol{\Gamma}$, a $\breve{\boldsymbol{\Gamma}}$-norm $\varphi: X \rightarrow \alpha$, and a cofinal function $\varrho: \omega^{\omega} \rightarrow \alpha$ witness that $A$ is of boundedness type. Let $\leq_{\varphi}$ be the prewellordering associated to $\varphi$.

By $\mathrm{pBI}-\mathrm{AD}$, we know that there is either a strongly optimal strategy for player I or for player II. Towards a contradiction, suppose that there is a strongly optimal strategy $\sigma$ for player I. 
Since $\varphi$ is a $\breve{\boldsymbol{\Gamma}}$-norm, the sets $U_{x}^{\leq \varphi, \mathrm{I}}$ are in $\boldsymbol{\Gamma}$ for all $x \in \omega^{\omega}$. Thus $B:=$ $\left\{\langle x, z\rangle ; \mu_{\sigma, z}^{-}\left(U_{x}^{\leq \varphi, \mathrm{I}}\right)>0\right\}$ is in $\boldsymbol{\Gamma}$ by $(\mathrm{KT} 2)$. By $(\mathrm{KT} 1), \Psi_{\leq \varphi}^{\sigma, \mathrm{I}}\left(\omega^{\omega}\right)=\exists^{\mathbb{R}} B \in \boldsymbol{\Gamma}$.

Now the Boundedness Lemma 2.1 gives us an ordinal $\beta<\alpha$ such that for all $y \in \Psi_{\leq \varphi}^{\sigma, \mathrm{I}}\left(\omega^{\omega}\right)$ we have $\varphi(y)<\beta$. Since $\varrho$ was cofinal, let $z$ be any real such that $\varrho(z)>\beta$.

If $x \in \Psi_{\leq \varphi}^{\sigma, \mathrm{I}}(\{z\}) \subseteq B$, then $\varphi\left(x_{\mathrm{I}}\right)<\varrho\left(x_{\mathrm{II}}\right)=\varrho(z)$ by choice of $z$, so $x \notin A$. But this contradicts Proposition 3.4, so $\sigma$ can't be strongly optimal for player I.

Note that the proof of the Boundedness Lemma 3.6 showed that player I can't have a weakly optimal strategy, since we only needed information about the measures $\mu_{\sigma, z}$ (as opposed to all measures $\mu_{\sigma, \tau}$ ). This yields the the following corollary:

Corollary 3.7. Assume bIBI-AD. Let $A$ be of boundedness type. Then there is a weakly optimal strategy for player II.

3.2. Analytic Blackwell determinacy: An application. Tony Martin announced in [Ma98] a proof of sharps from Bl-Det $\left(\boldsymbol{\Pi}_{1}^{1}\right)$. The proof is unpublished, therefore we include it in this paper with Martin's permission. The proof given here is essentially Martin's original proof, but uses the language of the simulation technique developed by the present author for the applications in Section 4. Our analysis of Martin's proof shows that instead of $\operatorname{BI}-\operatorname{Det}\left(\boldsymbol{\Pi}_{1}^{1}\right)$ we only need bIBI-Det $\left(\boldsymbol{\Pi}_{1}^{1}\right)$.

Theorem 3.8 (Martin). Suppose that bIBI-Det $\left(\boldsymbol{\Pi}_{1}^{1}\right)$ holds. Then for all reals $x$ the sharp $x^{\#}$ exists.

Proof. As usual, we show the theorem for $x=0$ since it relativizes easily. We shall prove the theorem with Harrington's original proof (cf. [Ha78]) in mind.

We consider the following game: Player I must play a code for a countable ordinal $\alpha$; if player I succeeds then player II's play must code a model with domain $\omega$ that is an end extension of $\mathbf{L}_{\alpha}$. Denote by $A$ the set of winning plays for player I. This set is $\Pi_{1}^{1}$ and of boundedness type, hence by assumption blindfoldedly Blackwell determined, and by Corollary 3.7, player II has a weakly optimal strategy $\tau$. By Harrington's proof, it is enough to show that $\tau$ has the following property:

Let $\gamma<\alpha<\omega_{1}$ and $\beta<\omega_{1}$ be ordinals. If $b \in \mathbf{L}_{\beta}$ with $b \subseteq \gamma$, and $b$ codes a wellordering of $\gamma$ of order type $\alpha$, then for every $z \in \mathrm{WO}$ with $\|z\|=\beta$, we have $b \in \mathbf{L}_{\gamma+\omega \cdot 2}[z, \tau]$. 
Set $B:=\left(2^{\omega} \backslash A\right)_{\{z\}}^{\mathrm{II}}$. For every formula $\varphi$ and every natural number $m$, we define

$$
B^{\varphi, m}:=\left\{x ; x \in B \& \forall \delta<\gamma\left(\delta \in b \leftrightarrow \mathbf{L}_{\gamma+\omega}\left[x_{\mathrm{II}}\right] \models \varphi\left[x_{\mathrm{II}}, m, \delta\right]\right)\right\} .
$$

By the usual argument, we get $\bigcup_{\varphi, m} B^{\varphi, m}=B$, and thus know that at least one of the sets $B^{\varphi, m}$ must have positive measure. Fix this set $B^{*}:=B^{\varphi^{*}, m^{*}}$.

By the continuity property of Borel measures we know that we find an $s \in 2^{<\omega}$ such that the basic open set $[s]$ has positive measure and we have

$$
\mu_{z, \tau}\left(B^{*} \cap[s]\right)>\frac{1}{2} \cdot \mu_{z, \tau}([s]) .
$$

We now finish the proof by defining $b$ in $\mathbf{L}_{\gamma+\omega \cdot 2}[z, \tau]$ as follows:

$$
\begin{aligned}
& \delta \in b \Longleftrightarrow \mu_{z, \tau}\left(\left\{x ; \mathbf{L}_{\gamma+\omega}\left[x_{\mathrm{II}}\right]=\varphi^{*}\left[\delta, m^{*}, x_{\mathrm{II}}\right]\right\} \cap[s]\right)>\frac{1}{2} \cdot \mu_{z, \tau}([s]) . \\
& \text { " } \Rightarrow \text { "If } \delta \in b \text {, then } B^{*} \subseteq\left\{x ; \mathbf{L}_{\gamma+\omega}\left[x_{\mathrm{II}}\right] \models \varphi^{*}\left[\delta, m^{*}, x_{\mathrm{II}}\right]\right\} \text {, so the claim }
\end{aligned}
$$
follows from the choice of $s$.

" $\Leftarrow$ " If $\delta \notin b$, then we again invoke the choice of $s$ to see that more than half of the measure of $[s]$ is taken by reals $x$ that are in $B^{*}$, thus they can't satisfy $\mathbf{L}_{\gamma+\omega}\left[x_{\mathrm{II}}\right] \models \varphi^{*}\left[\delta, m^{*}, x_{\mathrm{II}}\right]$.

Consequently, at the $\boldsymbol{\Pi}_{1}^{1}$ level, all mentioned forms of determinacy are equivalent:

Corollary 3.9. The following are equivalent:

1) $\operatorname{Det}\left(\boldsymbol{\Pi}_{1}^{1}\right)$,

2) $\operatorname{BI}-\operatorname{Det}\left(\boldsymbol{\Pi}_{1}^{1}\right)$,

3) $\mathrm{pBI}-\operatorname{Det}\left(\boldsymbol{\Pi}_{1}^{1}\right)$,

4) bIBI-Det $\left(\boldsymbol{\Pi}_{1}^{1}\right)$, and

5) for all $x \in \omega^{\omega}, x^{\#}$ exists.

This result suggests looking for other pointclasses with this property. It is unknown whether similar theorems can be proved for other pointclasses. ${ }^{2}$

3.3. Another application. We show that under Blackwell determinacy no wellordered sequence of pairwise different Borel sets can have length $\omega_{2}$. This has been proved under the assumption of AD by Leo Harrington $[\mathbf{H a 7 8}$, Theorem 4.5]. Our proof follows Harrington's proof closely, so we shall only mention the changes necessary for the Blackwell situation.

\footnotetext{
${ }^{2}$ In [MaNeVe03, Theorem 5.1, Corollary 5.3, Theorem 5.4, Theorem 5.6], the authors show equivalence of perfect information Blackwell determinacy and standard determinacy for the pointclasses $\boldsymbol{\Delta}_{n}^{1}, \boldsymbol{\Pi}_{2 n}^{1}$, and $\partial^{(n)}\left(<\omega^{2}-\boldsymbol{\Pi}_{1}^{1}\right)$; cf. Theorem 2.7.
} 
Lemma 3.10. Assume bIBI-AD. Then for every sequence of sets of reals $\left\langle B_{\xi} ; \xi<\omega_{1}\right\rangle$ with $B_{\xi} \in \boldsymbol{\Sigma}_{\beta}^{0}$ for some fixed $\beta<\omega_{1}$ there are $\gamma_{0}$ and $\gamma_{1}$ such that $B_{\gamma_{0}}=B_{\gamma_{1}}$.

\section{Corollary 3.11. bIBI-AD implies $\neg$ AC.}

From Lemma 3.10 we can easily deduce the theorem modulo an assumption which will be proved later.

Theorem 3.12. Assume either bIBI-AD+ " $\aleph_{2}$ is regular" or BI-AD. (Corollary 4.17 will show that $\mathrm{BI}-\mathrm{AD}$ implies that $\aleph_{2}$ is a regular cardinal.) Then no wellordered sequence of pairwise different Borel sets has length $\omega_{2}$.

Proof of Lemma 3.10. The proof is essentially Harrington's AD-proof with modifications where necessary. In this proof we shall use notation rather loosely and identify the reals played in the game with the objects coded by them. For example, when player I plays a code $v$ for a pair $\langle\eta, T\rangle$ against the strategy $\tau$, we denote the product measure derived from this by $\mu_{\langle\eta, T\rangle, \tau}$ instead of $\mu_{v, \tau}$.

Fix a universal $\boldsymbol{\Sigma}_{\beta}^{0}$ set $C$ with Borel code $c$ and play the game with the following winning conditions:

Player I plays $\langle\eta, T\rangle$ where $T$ is a tree on $\omega$ and $\eta \in T$ with defined height $h_{T}(\eta) \in \omega_{1}$; player II plays $\langle b, w\rangle$ where $b=\left\langle b_{i} ; i \in \omega\right\rangle$ is a countable sequence of reals and $w \in \mathrm{WO}$. Player II wins if

$$
\|w\|>h_{T}(\eta) \&\left\{C_{b_{i}} ; i \in \omega\right\}=\left\{B_{\xi} ; \xi<\|w\|\right\} .
$$

This game is clearly of boundedness type, so by Corollary 3.7, there is a weakly optimal strategy $\tau$ for player II.

We fix some $\gamma>\beta$ with $\omega \cdot \gamma=\gamma$. We define a prewellordering

$$
x \leq y: \Longleftrightarrow x \text { codes }\langle b, w\rangle, y \operatorname{codes}\left\langle b^{*}, w^{*}\right\rangle \text {, and }\|w\| \leq\left\|w^{*}\right\| .
$$

Consider the set $X_{\gamma}:=\left\{x ; x\right.$ codes $\langle\eta, T\rangle$ with $\left.h_{T}(\eta)<\gamma\right\}$ which is a Borel set. Then $\left\{w ; \exists b \exists y \in \Psi_{\leq}^{\tau, \text { II }}\left(X_{\gamma}\right)(y=\langle b, w\rangle)\right\}$ is a $\Sigma_{1}^{1}$ subset of WO ( $\boldsymbol{\Sigma}_{1}^{1}$ is a Kechris-Tanaka pointclass), and thus it is bounded by some countable ordinal. Call that ordinal $\varrho$ and assume without loss of generality that $\varrho>\gamma$.

We shall now show that there is a $\xi<\varrho$ such that $B_{\xi}=B_{\varrho}$.

Suppose that the claim is false. Fix an enumeration of $\varrho=\left\{\xi_{i} ; i \in \omega\right\}$. Then for all natural numbers $i$, the symmetric difference $D_{i}:=B_{\xi_{i}} \triangle B_{\varrho}$ is nonempty, say $d_{i} \in D_{i}$. Define a function $f \in 2^{\omega}$ by

$$
f(i)=1: \Longleftrightarrow d_{i} \notin B_{\varrho},
$$


and take a tree $T$ to be $\mathbf{L}_{\varrho+\omega}[\tau, d, f, c]$-generic in the sense of $[\mathbf{H a 7 8}$, Definition 2.8]. There must be an $\eta \in T$ such that $h_{T}(\eta)=\varrho$ by definition of genericity. Then

$\left(\psi_{\eta, T}\right) \quad \mu_{\langle\eta, T\rangle, \tau}\left(\left\{\langle\eta, T\rangle *\langle b, w\rangle ; \exists i \forall j\left(d_{j} \in C_{b_{i}} \Longleftrightarrow f(j)=0\right)\right\}\right)=1$, since the condition is true for all winning plays $\langle b, w\rangle$ for player II against $\langle\eta, T\rangle$.

Using Steel forcing as in Harrington's original proof, we get $T^{\prime}$ with $\eta \in T^{\prime}$, $h_{T^{\prime}}(\eta)<\gamma$ and $\psi_{\eta, T^{\prime}}$. This means that

$$
Y:=\left\{\left\langle\eta, T^{\prime}\right\rangle *\langle b, w\rangle ; \exists i \forall j\left(d_{j} \in C_{b_{i}} \Longleftrightarrow f(j)=0\right)\right\}
$$

is a $\mu_{\left\langle\eta, T^{\prime}\right\rangle, \tau^{-}}$-measure 1 set. Since $h_{T^{\prime}}(\eta)<\gamma$, we know that $\left\langle\eta, T^{\prime}\right\rangle \in X_{\gamma}$, so we can get some $\langle b, w\rangle \in \Psi_{\leq}^{\tau, \mathrm{II}}\left(\left\{\left\langle\eta, T^{\prime}\right\rangle\right\}\right) \cap\left(A_{\left\{\left\langle\eta, T^{\prime}\right\rangle\right\}}^{\mathrm{II}} \cap Y\right)_{\mathrm{II}}$. In particular, $\|w\|<\varrho$. This is enough to derive a contradiction.

\section{Infinitary combinatorics under the assumption of Blackwell determinacy.}

4.1. Infinitary combinatorics under AD. We shall give a brief overview of the basic structure theory below $\Theta$ under AD. For a historical account of infinitary combinatorics under $A D$, we refer the reader to $[\mathbf{K a 9 4}$, Chapter $28]$ and $[\mathbf{L o ̈ 0 2} \mathbf{b}, \S 3.1]$. The reader can also find proofs or pointers to proofs there.

Definition 4.1. Let $\kappa$ be a cardinal. We say that $\kappa$ has the strong partition property if $\kappa \rightarrow(\kappa)^{\kappa}$ holds.

The strong partition property of any infinite cardinal severely violates the Axiom of Choice. Of the rich structure theory of $\omega_{1}$ under AD, the following two results will be of most interest to us:

Theorem 4.2 (Solovay's Lemma). Assume AD. Then for every $A \subseteq \omega_{1}$ there is a real $x \in \omega^{\omega}$ such that $A \in \mathbf{L}[x]$.

Theorem 4.3 (Martin). Assume AD. Then $\aleph_{1}$ has the strong partition property.

We shall use the following convention: If $U$ is a normal $\sigma$-complete ultrafilter on an ordinal $\alpha$ and $\beta$ is another ordinal, then $\beta^{\alpha} / U$ is a well-ordered structure (using DC). We shall identify this structure with its ordertype.

Definition 4.4. Let $\kappa$ be a cardinal with the strong partition property and $\mu$ a normal measure on $\kappa$. We then define a sequence of well-ordered structures $\left\langle\kappa_{n}^{\mu} ; n \leq \omega\right\rangle$ as follows:

- $\kappa_{1}^{\mu}:=\kappa$,

- $\kappa_{n+1}^{\mu}:=\left(\kappa_{n}^{\mu}\right)^{\kappa} / \mu$, and 
- $\kappa_{\omega}^{\mu}:=\sup \left\{\kappa_{n}^{\mu} ; n \in \omega\right\}$.

This sequence is called the Kleinberg sequence derived from $\mu$.

Theorem 4.5 (Kleinberg). Let $\kappa$ be a cardinal with the strong partition property, $\mu$ be a normal ultrafilter on $\kappa$, and $\left\langle\kappa_{i}^{\mu} ; i \leq \omega\right\rangle$ the derived Kleinberg sequence. Then:

1) For all natural numbers $n \in \omega, \kappa_{n}^{\mu}<\kappa_{n+1}^{\mu}$,

2) $\kappa_{1}^{\mu}$ and $\kappa_{2}^{\mu}$ are measurable,

3) for all $n \geq 2, \operatorname{cf}\left(\kappa_{n}^{\mu}\right)=\kappa_{2}^{\mu}$,

4) for all $n \geq 3, \kappa_{n}^{\mu}$ is a Jónsson cardinal, and

5) $\sup \left\{\kappa_{n}^{\mu} ; n \in \omega\right\}$ is a Rowbottom cardinal.

Moreover, if $\kappa^{\kappa} / U=\kappa^{+}$, then $\kappa_{n+1}^{\mu}=\left(\kappa_{n}^{\mu}\right)^{+}$for all $n \in \omega$.

Corollary 4.6. Assume AD. Then $\aleph_{1}$ and $\aleph_{2}$ are measurable, ${ }^{3} \aleph_{n}$ for $3 \leq n<\omega$ is Jónsson, and $\aleph_{\omega}$ is Rowbottom.

Let $\lambda<\kappa$ be regular cardinals. Let us denote by $\mathcal{C}_{\kappa}$ the closed unbounded filter on $\kappa$. Then we define

$$
\mathcal{C}_{\kappa}^{\lambda}:=\left\{X \subseteq \kappa ; \exists C \in \mathcal{C}_{\kappa}(C \cap\{\xi<\kappa ; \operatorname{cf}(\xi)=\lambda\} \subseteq X)\right\} .
$$

We furthermore define the projective ordinals by $\boldsymbol{\delta}_{n}^{1}:=\sup \left\{\xi ; \xi\right.$ is the length of a prewellordering of $\omega^{\omega}$ in $\left.\boldsymbol{\Delta}_{n}^{1}\right\}$.

Fact 4.7. Let $n$ be a natural number. Assume AD. Then:

1) (Kunen, Martin 1971) $\boldsymbol{\delta}_{2 n+2}^{1}=\left(\boldsymbol{\delta}_{2 n+1}^{1}\right)^{+}$,

2) (Kechris 1974) $\boldsymbol{\delta}_{2 n+1}^{1}$ is the cardinal successor of a cardinal of cofinality $\omega$

3) (Martin, Kunen 1971) all $\boldsymbol{\delta}_{n}^{1}$ are measurable,

4) (Martin, Kunen 1971) $\boldsymbol{\delta}_{1}^{1}=\aleph_{1}, \boldsymbol{\delta}_{2}^{1}=\aleph_{2}, \boldsymbol{\delta}_{3}^{1}=\aleph_{\omega+1}$, and $\boldsymbol{\delta}_{4}^{1}=\aleph_{\omega+2}$,

5) (Martin, Paris 1971) $\boldsymbol{\delta}_{1}^{1} \rightarrow\left(\boldsymbol{\delta}_{1}^{1}\right)^{\boldsymbol{\delta}_{1}^{1}}$, and for all $\alpha<\boldsymbol{\delta}_{2}^{1}$, the relation $\boldsymbol{\delta}_{2}^{1} \rightarrow\left(\boldsymbol{\delta}_{2}^{1}\right)^{\alpha}$ holds

6) (Martin 1971) for all $\alpha<\omega_{1}$ the partition relation $\boldsymbol{\delta}_{2 n+1}^{1} \rightarrow\left(\boldsymbol{\delta}_{2 n+1}^{1}\right)^{\alpha}$ holds,

7) (Kunen 1971) the $\omega$-cofinal measure $\mathcal{C}_{\boldsymbol{\delta}_{2 n+1}^{1}}^{\omega}$ is a normal measure on $\boldsymbol{\delta}_{2 n+1}^{1}$ with $\boldsymbol{\delta}_{2 n+1}^{1} \boldsymbol{\delta}_{2 n+1}^{1} / \mathcal{C}_{\boldsymbol{\delta}_{2 n+1}^{1}}^{\omega}=\boldsymbol{\delta}_{2 n+2}^{1}$, and

8) (Martin 1980) $\boldsymbol{\delta}_{3}^{1} \boldsymbol{\delta}_{3}^{1} / \mathcal{C}_{\boldsymbol{\delta}_{3}^{1}}^{\omega_{1}}=\aleph_{\omega \cdot 2+1}$ and $\boldsymbol{\delta}_{3}^{1} \boldsymbol{\delta}_{3}^{1} / \mathcal{C}_{\boldsymbol{\delta}_{3}^{1}}^{\omega_{2}}=\aleph_{\omega^{\omega}+1}$, and these two cardinals are measurable.

9) (Jackson 1983) Let $E$ be the function recursively defined by $E(1)=0$ and $E(n+1)=\omega^{E(n)}$. Then for every $n \in \omega$,

$$
\boldsymbol{\delta}_{2 n+1}^{1}=\aleph_{E(2 n+1)+1},
$$

\footnotetext{
${ }^{3}$ The measurability of $\aleph_{2}$ had been proved by Solovay long before that using ideas of Tony Martin [Ka94, Theorem 28.6].
} 
and all odd projective ordinals have the strong partition property.

4.2. The combinatorial theory of $\aleph_{1}$ under the assumption of Blackwell determinacy. In this section we shall prove that the combinatorial theory of $\aleph_{1}$ behaves under Blackwell determinacy axioms as it does under $A D$ in many respects. We start with the Blackwell analogue of Solovay's Lemma 4.2.

Theorem 4.8. Assume bIBI-AD. Then for every $Y \subseteq \omega_{1}$ there is an $a \in \omega^{\omega}$ and a formula $\vartheta$ such that

$$
\xi \in Y \Longleftrightarrow \mathbf{L}[a] \models \vartheta[\xi, a] .
$$

Proof. The proof follows Solovay's original proof idea closely; cf. [Ka94, Theorem 28.5].

We look at the following game: Given $Y \subseteq \omega_{1}$, player I plays an ordinal $\alpha$ and player II tries to play $Y \cap \beta$ for some $\beta>\alpha$. Obviously, $A$ is of boundedness type. Thus by Corollary 3.7, we have a weakly optimal strategy $\tau$ for player II.

For each $z \in \mathrm{WO}$, we let $B_{z}:=\left\{y ; y_{\mathrm{I}}=z \&\right.$ there is an $i \in \omega$ such that $\left\langle\omega, E_{y_{\mathrm{I}}}\right\rangle$ and $\left\langle\omega, E_{\left(y_{\mathrm{II}}\right)_{i}}\right\rangle$ are isomorphic $\}$.

Since the sets $B_{z}$ are $\boldsymbol{\Sigma}_{1}^{1}$, and $\boldsymbol{\Sigma}_{1}^{1}$ is a Kechris-Tanaka pointclass by Theorem 3.2 , we have that

$\varphi\left(v_{0}, v_{1}\right): \Longleftrightarrow v_{0} \in \mathrm{WO} \& v_{1}$ is a strategy for player II $\& \mu_{v_{0}, v_{1}}\left(B_{v_{0}}\right)>\varepsilon$ is $2-\Pi_{1}^{1}$ (in the Hausdorff difference hierarchy). By Shoenfield's absoluteness lemma, $2-\Pi_{1}^{1}$ relations are absolute.

We let $\mathbb{P}_{\xi}$ denote the forcing partial order adding a bijection from $\omega$ to $\xi$, and $\dot{z}_{\xi}$ be a name for this bijection (defined uniformly in $\xi$ ). Then the formula $\vartheta\left(v_{0}, v_{1}\right)$ saying " $v_{0}$ is an ordinal, $v_{1}$ is a strategy for player II, and $\Vdash_{\mathbb{P}_{v_{0}}} \varphi\left({\dot{z_{0}}}_{v_{0}}, \check{v_{1}}\right)$ " proves the theorem as in Solovay's original proof.

Corollary 4.9. Assume bIBI-AD. Then:

1) For every $Y \subseteq \omega_{1}$ there is a real $a \in \omega^{\omega}$ such that $Y \in \mathbf{L}[a]$, and

2) $\boldsymbol{\delta}_{2}^{1}=\aleph_{2}$, and

3) there is an inner model with a strong cardinal.

Proof. The first two claims are standard (note that the second claim uses the existence of sharps which we get from Corollary 3.9). As to the third, sharps plus " $\delta_{2}^{1}=\aleph_{2}$ " give an inner model with a strong cardinal by a theorem of Steel and Welch [StWe98, Theorem 3.9].

Actually, the methods of Steel and Welch allow to go a bit beyond a strong cardinal. Even beyond that, if we allow a measurable cardinal in our metatheoretical assumptions, Hjorth claims that the validity of Solovay's Lemma alone implies the existence of an inner model with a Woodin cardinal (personal communication, 2001). 
4.3. A general technique to prove infinite partition relations. We shall now develop the analogue of a general technique of proving infinitary partition relations. The technique is in essence due to Martin. Terminology and notation are due to Kechris [Ke78]:

Definition 4.10. Let $\lambda \leq \kappa$ be ordinals. We call a family of objects

$$
\mathcal{C}:=\left\langle C_{\xi}, C_{\xi, \vartheta}, f^{\xi} ; \xi<\omega \cdot \lambda, \vartheta<\kappa\right\rangle
$$

a $\langle\lambda, \kappa\rangle$-Martin system if it satisfies the following conditions:

(M1) For every $\xi<\omega \cdot \lambda$ and every $\vartheta<\kappa, C_{\xi}$ and $C_{\xi, \vartheta}$ are sets of reals.

(M2) For every $\xi<\omega \cdot \lambda, f^{\xi}: C_{\xi} \rightarrow \kappa$ is a function.

(M3) For every $\xi<\omega \cdot \lambda$ and every $\vartheta<\kappa$, we have that $C_{\xi, \vartheta} \subseteq \bigcap_{\eta \leq \xi} C_{\eta}$.

For any given Martin system $\mathcal{C}$, we shall use the following notation:

The set $\operatorname{core}(\mathcal{C}):=\bigcap_{\xi \in \omega \cdot \lambda} C_{\xi}$ will be called the core of $\mathcal{C}$. For each element $x$ of the core, we can define the function $f_{x} \in[\kappa]^{\omega \cdot \lambda}$ by $f_{x}(\xi):=$ $f^{\xi}(x) .{ }^{4}$ For any continuous function $\sigma: \omega^{\omega} \rightarrow \omega^{\omega}$, we define

$$
i_{\mathcal{C}}^{\sigma}(\xi, \vartheta):=\sup \left\{f^{\xi}(x)+1 ; x \in \sigma " C_{\xi, \vartheta}\right\} .
$$

Definition 4.11. Let $\mathcal{C}$ be any $\langle\kappa, \lambda\rangle$-Martin system. We call $\mathcal{C}$ perfectly good if the following conditions hold:

(P1) For any $f \in[\kappa]^{\omega \cdot \lambda}$ there is an $x \in \operatorname{core}(\mathcal{C}) \cap \bigcap_{\xi<\omega \cdot \lambda} C_{\xi, f(\xi)}$ such that $f_{x}=f$.

(P2) For any continuous function $\sigma: \omega^{\omega} \rightarrow \omega^{\omega}$ with the property

$$
\sigma " \bigcap_{\eta \leq \xi} C_{\eta} \subseteq C_{\xi}
$$

we have $i_{\mathcal{C}}^{\sigma}(\xi, \vartheta)<\kappa$.

The existence of a perfectly good Martin system gives rise to an abstract proof of Martin's Theorem 4.3:

Theorem 4.12 (Martin). Let $\lambda \leq \kappa$ be ordinals, $\kappa$ a regular cardinal, and suppose that there is a perfectly good $\langle\lambda, \kappa\rangle$-Martin system. Then AD implies that $\kappa \rightarrow(\kappa)^{\lambda}$ holds. (Cf. [Ke78, Lemma 11.1].)

Using the simulation technique, we define the Blackwell analogue of this notion:

\footnotetext{
${ }^{4}$ We extend the functions $f^{\xi}$ to $\omega^{\omega}$ by
}

$$
f^{\xi}(x):=\left\{\begin{array}{cl}
f^{\xi}(x) & \text { if } x \in C_{\xi} \\
\infty & \text { otherwise. }
\end{array}\right.
$$

In the following we shall not distinguish between the functions $f^{\xi}: C_{\xi} \rightarrow \kappa$ and $f^{\xi}: \omega^{\omega} \rightarrow$ $\kappa \cup\{\infty\}$. 
Fixing any Martin system $\mathcal{C}$ we define the prewellordering

$$
\begin{gathered}
x \leq_{\xi} y: \Longleftrightarrow f^{\xi}(x) \leq f^{\xi}(y) \vee y \notin C_{\xi} \text {, and the functions } \\
\psi_{\mathcal{C}}^{\sigma}(\xi, \vartheta):=\sup \left\{f^{\xi}(x)+1 ; x \in \Psi_{\leq_{\xi}, \mathrm{I}}^{\sigma,}\left(C_{\xi, \vartheta}\right)\right\}, \text { and } \\
\psi_{\mathcal{C}}^{\tau}(\xi, \vartheta):=\sup \left\{f^{\xi}(x)+1 ; x \in \Psi_{\leq \xi}^{\tau, \mathrm{II}}\left(C_{\xi, \vartheta}\right)\right\} .
\end{gathered}
$$

We shall call a mixed strategy $\sigma$ for player I $\mathcal{C}$-adequate if for all $\xi<\omega \cdot \lambda$, the following holds:

$$
\Psi_{\leq \xi}^{\sigma, \mathrm{I}}\left(\bigcap_{\eta \leq \xi} C_{\eta}\right) \subseteq C_{\xi} .
$$

Similarly, we call a mixed strategy $\tau$ for player II $\mathcal{C}$-adequate if for all $\xi<\omega \cdot \lambda$, we have:

$$
\Psi_{\leq \xi}^{\tau, \mathrm{II}}\left(\bigcap_{\eta \leq \xi} C_{\eta}\right) \subseteq C_{\xi} .
$$

Now we can extend Definition 4.11 naturally to the Blackwell context:

Definition 4.13. Let $\mathcal{C}$ be any $\langle\kappa, \lambda\rangle$-Martin system. We call $\mathcal{C}$ imperfectly good if it satisfies (P1) and:

(I2) For any adequate strategy $\sigma$ and any $\xi<\omega \cdot \lambda$ and $\vartheta<\kappa$, we have $\psi_{\mathcal{C}}^{\sigma}(\xi, \vartheta)<\kappa$.

Note that by $(\mathrm{P} 1)$, if $\mathcal{C}$ is an imperfectly good Martin system, then for all $\xi, f^{\xi} " C_{\xi} \supseteq\{\nu ; \xi \leq \nu\}$ holds.

Theorem 4.14 (Abstract Martin Theorem). Let $\lambda \leq \kappa$ be ordinals, $\kappa$ a regular cardinal in $\mathbf{L}(\mathbb{R})$ and suppose that there is an imperfectly good $\langle\lambda, \kappa\rangle$ Martin system in $\mathbf{L}(\mathbb{R})$. Then $\mathrm{BI}-\mathrm{AD}$ implies that $\kappa \rightarrow(\kappa)^{\lambda}$ holds. (If $\lambda<\omega_{1}$, we only need bIBI-AD.)

Proof. In this proof we shall mostly be using only bIBI-AD. There is only a single point in the proof where we have to use Theorem 2.8 if $\lambda$ is uncountable. The proof follows Martin's proof closely: We fix an imperfectly good Martin system $\mathcal{C}=\left\langle C_{\xi}, C_{\xi, \vartheta}, f^{\xi} ; \xi<\omega \cdot \lambda, \vartheta<\kappa\right\rangle$ and a partition $a:[\kappa]^{\lambda} \rightarrow 2$. We want to show that there is an $a$-homogeneous set. As in the usual proof, we look at the following game:

Given a real $x$ such that $x_{\mathrm{I}}, x_{\mathrm{II}} \in \operatorname{core}(\mathcal{C})$, define $p_{x}: \lambda \rightarrow \kappa$ by

$$
p_{x}(\vartheta):=\max \left(\sup \left\{f_{x_{\mathrm{I}}}(\omega \cdot \vartheta+n) ; n \in \omega\right\}, \sup \left\{f_{x_{\mathrm{II}}}(\omega \cdot \vartheta+n) ; n \in \omega\right\}\right) .
$$

Now set

$$
\begin{gathered}
x \in A: \Longleftrightarrow \quad \exists \xi<\omega \cdot \lambda\left(x_{\mathrm{II}} \notin C_{\xi} \& \forall \zeta \leq \xi\left(x_{\mathrm{I}} \in C_{\zeta}\right)\right) \\
\vee\left(\forall \xi<\omega \cdot \lambda\left(x_{\mathrm{I}} \in C_{\xi} \& x_{\mathrm{II}} \in C_{\xi}\right) \&\right. \\
\left.p_{x} \in[\kappa]^{\lambda} \& a\left(p_{x}\right)=0\right) .
\end{gathered}
$$


We shall show that a weakly optimal strategy $\sigma$ for player I in the game with payoff $A$ gives an $a$-homogeneous set for the value 0 (and similarly a weakly optimal strategy for player II gives an $a$-homogeneous set for the value 1$)$.

Claim 1. $\sigma$ is $\mathcal{C}$-adequate.

Proof. Take any $x \in \Psi_{\leq \xi}^{\sigma, \mathrm{I}}\left(\bigcap_{\eta \leq \xi} C_{\eta}\right)$ and $z$ witnessing this, i.e., $\mu_{\sigma, z}^{-}\left(U_{x}^{\leq \xi, \mathrm{I}}\right)>$ 0 . By definition, $z \in \bigcap_{\eta \leq \xi} C_{\eta}$. Towards a contradiction, suppose that $x_{\mathrm{I}} \notin$ $C_{\xi}$. Then $U:=\left(U_{x}^{\leq \xi}, \mathrm{I}\right)_{\{z\}}^{\mathrm{I}}$ is disjoint from $A$ : Every element of $U$ represents a run of the game where player II plays into every $C_{\eta}$ for $\eta \leq \xi$, but player I doesn't play into $C_{\xi}$, hence player I loses. But $\mu_{\sigma, z}^{-}(U)=\mu_{\sigma, z}^{-}\left(U_{x}^{\leq \xi}, \mathrm{I}\right)>0$, contradicting the weak optimality of $\sigma$.

With Claim 1 we know by (I2) that $\psi_{\mathcal{C}}^{\sigma}(\xi, \vartheta)<\kappa$ for all $\xi<\omega \cdot \lambda$ and $\vartheta<\kappa$.

Claim 2. Let $\xi<\omega \cdot \lambda$ and $\vartheta<\kappa$. Then for every $x \in C_{\xi, \vartheta}$ the set $\left\{u ; f^{\xi}\left(u_{\mathrm{I}}\right)<\psi_{\mathcal{C}}^{\sigma}(\xi, \vartheta)\right\}$ has $\mu_{\sigma, x}$-measure 1 .

Proof. Suppose not. Then there is an $x$ such that $\left\{u ; f^{\xi}\left(u_{\mathrm{I}}\right) \geq \psi_{\mathcal{C}}^{\sigma}(\xi, \vartheta)\right\}$ has positive $\mu_{\sigma, x}$-measure. Let $r$ be such that $f^{\xi}(r)=\psi_{\mathcal{C}}^{\sigma}(\xi, \vartheta)$. Then $r \in \Psi_{\leq_{\xi}}^{\sigma, \mathrm{I}}\left(C_{\xi, \vartheta}\right)$. But then $f^{\xi}(r)<\psi_{\mathcal{C}}^{\sigma}(\xi, \vartheta)$ by the definition of $\psi_{\mathcal{C}}^{\sigma}$.

The set

$$
D:=\left\{\varrho ; \forall \xi<\omega \cdot \lambda \forall \vartheta<\kappa\left(\xi<\varrho \& \vartheta<\varrho \rightarrow \psi_{\mathcal{C}}^{\sigma}(\xi, \vartheta)<\varrho\right)\right\}
$$

is a closed unbounded set in $\kappa$ (using the assumptions that $\kappa$ is regular in $\mathbf{L}(\mathbb{R})$ and that the Martin system $\mathcal{C}$ was in $\mathbf{L}(\mathbb{R})$ ). As in the classical proof, let

$$
[D]_{u}^{\lambda}:=\left\{g \in[D]^{\lambda} ; \exists f \in[\kappa]^{\omega \cdot \lambda} \forall \vartheta<\lambda\left(g(\vartheta)=\sup _{n \in \omega} f(\omega \cdot \vartheta+n)\right)\right\} .
$$

It is enough to show $a "[D]_{u}^{\lambda}=\{0\}$ to complete the proof.

Pick any $g \in[D]_{u}^{\lambda}$. This fact is witnessed by some $f \in[\kappa]^{\omega \cdot \lambda}$ such that $g(\vartheta)=\sup _{n} f(\omega \cdot \vartheta+n)$. By Property (P1), we find an element $z \in \bigcap_{\xi<\omega \cdot \lambda} C_{\xi, f(\xi)}$ with $f_{z}=f$. We let player II play this $z$ and set $A^{*}:=A_{\{z\}}^{\mathrm{I}}$. If $U_{\xi}:=\left\{u ; f^{\xi}\left(u_{\mathrm{I}}\right)<\psi_{\mathcal{C}}^{\sigma}(\xi, f(\xi))\right\}$, then Claim 2 tells us that $\mu_{\sigma, z}^{-}\left(U_{\xi}\right)=1$. By Theorem $2.8,{ }^{5}$ we get

$$
\mu_{\sigma, z}^{-}\left(A^{*} \cap \bigcap_{\xi<\omega \cdot \lambda} U_{\xi}\right)=1,
$$

${ }^{5}$ If $\lambda<\omega_{1}, \sigma$-completeness of the measure suffices, and we don't need to invoke BI-AD here. 
in particular, this set is nonempty. We pick any $w \in A^{*} \cap \bigcap_{\xi<\omega \cdot \lambda} U_{\xi}$, set $x:=w * z$ and finish the proof as in the classical proof by proving $g=p_{x}$, and thus $a(g)=0$ because $x \in A$.

With the Abstract Martin Theorem 4.14 at hand, we can reproduce part of the structure theory under AD under the assumption of BI-AD.

Theorem 4.15. BI-AD implies that $\omega_{1} \rightarrow\left(\omega_{1}\right)^{\omega_{1}}$ holds.

Proof. By Theorem 4.14, we just have to show that there is an imperfectly good $\left\langle\omega_{1}, \omega_{1}\right\rangle$-Martin system in $\mathbf{L}(\mathbb{R})$. The system is defined exactly as in the perfect information case (cf. $[$ Ke78] $){ }^{6}$

For $\xi, \vartheta<\omega_{1}$, we define:

$$
\begin{aligned}
C_{\xi}:=\left\{x ; \operatorname{EMB}\left(x^{+}\right) \& \xi, t_{x(0)}^{\mathcal{M}\left(x^{+}, \xi+\omega\right)}[\xi] \in \operatorname{wfp}\left(\mathcal{M}\left(x^{+}, \xi+\omega\right)\right)\right\}, \\
f^{\xi}(x):=t_{x(0)}^{\mathcal{M}\left(x^{+}, \xi+\omega\right)}[\xi], \text { and } \\
C_{\xi, \vartheta}:=\left\{x ; \forall \xi^{*} \leq \xi \exists \vartheta^{*} \leq \vartheta\left(x \in C_{\xi^{*}} \& t_{x(0)}^{\mathcal{M}\left(x^{+}, \xi^{*}+\omega\right)}\left[\xi^{*}\right] \leq \vartheta^{*}\right)\right\} .
\end{aligned}
$$

That $\mathcal{C}$ is a Martin system is immediate from the definitions. It is also clear that it is in $\mathbf{L}(\mathbb{R})$. ( $\mathrm{P} 1)$ is the same as in the classical case, so we only have to show (I2) (we only show it for strategies for player I):

First of all, notice that $C_{\xi, \vartheta}$ is Borel. Since $\leq_{\xi}$ is a $\boldsymbol{\Pi}_{1}^{1}$ norm, $U_{x}^{\leq_{\xi}, \mathrm{I}}$ is $\boldsymbol{\Sigma}_{1}^{1}$. We can combine Theorem 3.2 and Proposition 3.3 to get that $\Psi_{\leq \xi}^{\sigma, \mathrm{I}}\left(C_{\xi, \vartheta}\right)$ is $\boldsymbol{\Sigma}_{1}^{1}$.

We define a function $e_{\xi}: C_{\xi} \rightarrow \mathrm{WO}$ as follows:

For $x \in C_{\xi}$, let $E_{x, \xi}$ be the binary relation of the model $\mathcal{M}\left(x^{+}, \xi+\omega\right)$ and

$$
S_{x, \xi}:=\left\{n \in \omega ; \exists N\left(\mathcal{M}\left(x^{+}, \xi+\omega\right) \models N=t_{x(0)}[\xi] \& n E_{x, \xi} N\right)\right\} .
$$

Let $s_{x, \xi}: \omega \rightarrow S_{x, \xi}$ be the increasing enumeration of $S_{x, \xi}$. Then we set

$$
e_{\xi}(x)(\ulcorner k, \ell\urcorner)=1: \Longleftrightarrow s_{x, \xi}(k) E_{x, \xi} s_{x, \xi}(\ell) .
$$

Obviously, $e_{\xi}(x)$ is a code for the ordinal representing $t_{x(0)}^{\mathcal{M}\left(x^{+}, \xi+\omega\right)}[\xi]$. Thus $\left\|e_{\xi}(x)\right\|=f^{\xi}(x)$.

\footnotetext{
${ }^{6}$ We shall be using Kanamori's notation for Ehrenfeucht-Mostowski blueprints and related objects from $\left[\mathbf{K a 9 4}, \S 9 \&\right.$ p. 393]: We have formulae $\operatorname{EMB}\left(v_{0}\right)$ and $\operatorname{WF}\left(v_{0}\right)$ such that the following are equivalent:

1) $x$ is a remarkable, wellfounded Ehrenfeucht-Mostowski blueprint, and

2) $\operatorname{EMB}(x)$ and $\operatorname{WF}(x)$.

The formula $\operatorname{WF}\left(v_{0}\right)$ says that if the models $\mathcal{M}\left(\alpha, v_{0}\right)$ (defined in [Ka94, Lemma 9.4]) exist, then they are wellfounded for all $\alpha$. Most importantly, the formula EMB is an arithmetic formula. $\left\langle t_{n} ; n \in \omega\right\rangle$ is a list of all terms with one free variable denoting ordinals, so if $M$ is a model and $\xi \in M$ is an ordinal, then $t_{n}^{M}[\xi]$ is an $M$-ordinal. For a (not necessarily wellfounded) model $M, \operatorname{wfp}(M)$ denotes the wellfounded part of $M$. We shall also be using the notation $x^{+}(n):=x(n+1)$.
} 
If $\sigma$ is adequate, we can apply $e_{\xi}$ to the inequality

$$
\Psi_{\leq \xi}^{\sigma, \mathrm{I}}\left(C_{\xi, \vartheta}\right) \subseteq C_{\xi}
$$

and receive

$$
e_{\xi} "\left(\Psi_{\leq \xi}^{\sigma, \mathrm{I}}\left(C_{\xi, \vartheta}\right)\right) \subseteq \mathrm{WO}
$$

The left-hand side is a $\boldsymbol{\Sigma}_{1}^{1}$ set, thus by boundedness, it is bounded in WO, say, by $\zeta<\omega_{1}$. But this means that $f^{\xi} "\left(\Psi_{\leq \xi}^{\sigma, \mathrm{I}}\left(C_{\xi, \vartheta}\right)\right)$ is bounded by $\zeta$ which was to be shown.

Kleinberg's Theorem 4.5 now gives us a Kleinberg sequence starting from $\aleph_{1}$. We can determine their exact values with the the "moreover" part of Theorem 4.5 holds which follows from the following theorem (for a proof, cf. $[\mathbf{K} 177])$ :

Theorem 4.16 (Solovay). Suppose that:

1) For every real there is a sharp,

2) for every subset $X \subseteq \omega_{1}$ there is a formula $\vartheta$ and a real a such that

$$
\xi \in X \Longleftrightarrow \mathbf{L}[a]=\vartheta[\xi, a] \text {, and }
$$

3) the closed unbounded filter on $\aleph_{1}$ is a normal ultrafilter. Then $\omega_{1}^{\omega_{1}} / \mathcal{C}_{\omega_{1}}^{\omega}=\omega_{2}$. Moreover, $\mathcal{C}_{\omega_{1}}^{\omega}$ is a canonical measure.

Corollary 4.17. Assume BI-AD. Then $\aleph_{1}$ and $\aleph_{2}$ are measurable, $\aleph_{n}$ is Jónsson $($ for $3 \leq n<\omega)$ and $\aleph_{\omega}$ is Rowbottom.

We now move to look at the odd partition ordinals $\boldsymbol{\delta}_{2 n+1}^{1}$ :

Proposition 4.18. Assume pBI-AD. Then $\boldsymbol{\delta}_{2 n+1}^{1} \rightarrow\left(\boldsymbol{\delta}_{2 n+1}^{1}\right)^{\lambda}$ for all $\lambda<$ $\omega_{1}$.

Proof. Since $\lambda<\omega_{1}$, we can fix a coding of sequences of reals of length $\omega \cdot \lambda$. Let $(\cdot)_{\xi}: \omega^{\omega} \rightarrow \omega^{\omega}$ be the $\xi$ th component map of this coding. Let $W$ be a complete $\boldsymbol{\Pi}_{2 n+1}^{1}$ set with $\boldsymbol{\Pi}_{2 n+1}^{1}$ norm $\varphi$ onto $\boldsymbol{\delta}_{2 n+1}^{1}$.

Following [Ke78, Theorem 11.2], we define for $\xi<\omega \cdot \lambda$ and $\vartheta<\boldsymbol{\delta}_{2 n+1}^{1}$

$$
\begin{gathered}
C_{\xi}:=\{x ; \varphi(x) \in W\}, \\
f^{\xi}(x):=\varphi\left((x)_{\xi}\right), \text { and } \\
C_{\xi, \vartheta}:=\left\{x ; \forall \xi^{*} \leq \xi \exists \vartheta^{*} \leq \vartheta\left((x)_{\vartheta^{*}} \in W \& \varphi\left((x)_{\xi^{*}}\right) \leq \vartheta^{*}\right)\right\} .
\end{gathered}
$$

With Theorem 4.14, we only have to show that

$$
\mathcal{C}:=\left\langle C_{\xi}, C_{\xi, \vartheta}, f^{\xi} ; \xi<\omega \cdot \lambda, \vartheta<\boldsymbol{\delta}_{2 n+1}^{1}\right\rangle
$$

is an imperfectly good Martin system. Note that since $\lambda<\omega_{1}$, we can apply Theorem 4.14 without BI-AD.

Again, it is obvious that $\mathcal{C}$ is a Martin system in $\mathbf{L}(\mathbb{R})$. We use pBI-AD to employ the Martin-Neeman-Vervoort Equivalence Theorem 2.7 and get 
$(A D)^{\mathbf{L}(\mathbb{R})}$. The projective ordinals are absolute between $\mathbf{V}$ and $\mathbf{L}(\mathbb{R})$, so we get that $\boldsymbol{\delta}_{n}^{1}=\left(\boldsymbol{\delta}_{n}^{1}\right)^{\mathbf{L}(\mathbb{R})}$ is regular in $\mathbf{L}(\mathbb{R})$ from $(\mathrm{AD})^{\mathbf{L}(\mathbb{R})}$ and Fact 4.7 (3). Again, (P1) doesn't have to be shown, leaving only (I2) to be checked:

Without loss of generality we show (I2) just for strategies of player I. Because $\varphi$ was a $\boldsymbol{\Pi}_{2 n+1}^{1}$-norm, the sets $C_{\xi, \vartheta}$ and $U_{x}^{\leq \xi, \text { I }}$ are $\boldsymbol{\Delta}_{2 n+1}^{1}$. Since $\boldsymbol{\Sigma}_{2 n+1}^{1}$ is a Kechris-Tanaka pointclass, the sets $\Psi_{\leq \xi}^{\sigma, \mathrm{I}}\left(C_{\xi, \vartheta}\right)$ are $\boldsymbol{\Sigma}_{2 n+1}^{1}$. If $\sigma$ is adequate, we have $\left\{(x)_{\xi} ; x \in \Psi_{\leq_{\xi}}^{\sigma, \mathrm{I}}\left(C_{\xi, \vartheta}\right)\right\}$ is a $\boldsymbol{\Sigma}_{2 n+1}^{1}$ subset of $W$, hence bounded by Theorem 2.1, and thus $\psi^{\sigma}(\xi, \vartheta)<\boldsymbol{\delta}_{2 n+1}^{1}$.

Corollary 4.19. Assume pBI-AD. Then all odd projective ordinals $\boldsymbol{\delta}_{2 n+1}^{1}$ are measurable.

Corollary 4.20. Assume BI-AD. Then $\boldsymbol{\delta}_{3}^{1}=\aleph_{\omega+1}$.

Proof. By the Martin-Neeman-Vervoort Equivalence Theorem 2.7 we know that PD holds whence (by a result of Kechris and Moschovakis [Ke78, Theorem $9.1(5)]$ ) the projective ordinals are strictly increasing and in particular $\boldsymbol{\delta}_{3}^{1}>\boldsymbol{\delta}_{2}^{1}=\aleph_{2}$ (using Corollary 4.9).

By Corollary $4.19, \delta_{3}^{1}$ is regular. Using Theorem 4.5, we know the cofinalities below $\aleph_{\omega+1}$, since $\left\langle\aleph_{n} ; n \geq 1\right\rangle$ is a Kleinberg sequence: $\operatorname{cf}\left(\aleph_{n}\right)=\aleph_{2}$ (for $n \geq 3$ ) and $\operatorname{cf}\left(\aleph_{\omega}\right)=\aleph_{0}$. So, $\boldsymbol{\delta}_{3}^{1} \geq \aleph_{\omega+1}$. Since by Theorem 3.8 sharps for reals exist, we can employ the Martin-Solovay analysis of $\Sigma_{3}^{1}$ sets under the existence of sharps (cf. [Ke78, Theorem 6.3]) and get $\boldsymbol{\delta}_{3}^{1} \leq \aleph_{\omega+1}$. This proves the claim.

\section{Open problems.}

At the moment, we cannot say much on combinatorics on projective ordinals beyond $\boldsymbol{\delta}_{3}^{1}$. Even of the following consequences of AD for $\boldsymbol{\delta}_{3}^{1}$ it is unknown whether they hold under any sort of Axiom of Blackwell determinacy [listed from harder to easier]:

1) $\boldsymbol{\delta}_{3}^{1} \rightarrow\left(\boldsymbol{\delta}_{3}^{1}\right)^{1}$

2) $\boldsymbol{\delta}_{3}^{1} \rightarrow\left(\boldsymbol{\delta}_{3}^{1}\right)^{\lambda}$ for all $\lambda<\boldsymbol{\delta}_{3}^{1}$,

3) $\mathcal{C}_{\boldsymbol{\delta}_{3}^{1}}^{1}$ is a normal ultrafilter on $\boldsymbol{\delta}_{3}^{1}$,

4) $\mathcal{C}_{\boldsymbol{\delta}_{3}^{1}}^{\boldsymbol{\delta}_{1}^{1}}$ is a normal ultrafilter on $\boldsymbol{\delta}_{3}^{1}$.

Even less is know about the $\boldsymbol{\delta}_{n}^{1}$ for $n>3$. It is not even known that $\delta_{4}^{1} \geq \aleph_{\omega+2}$. For all we know, it could be an ordinal between $\aleph_{\omega+1}$ and $\aleph_{\omega+2}$.

The reason for that is the lack of an analogue of the Moschovakis Coding Lemma.

In the following, we shall abbreviate with CL the statement of the Moschovakis Coding Lemma (cf. [Mo80, 7D.5]). 
Given the Martin-Neeman-Vervoort Equivalence Theorem 2.7, a proof of $\mathrm{CL}$ from Blackwell determinacy would immediately settle many of the questions on projective ordinals under Blackwell determinacy. For example, $\mathrm{PD}+\mathrm{CL}$ is enough to show that $\boldsymbol{\delta}_{n}^{1}$ is a cardinal, that $\boldsymbol{\delta}_{2 n+2}^{1}=\left(\boldsymbol{\delta}_{2 n+1}^{1}\right)^{+}$, and that $\boldsymbol{\delta}_{2 n+1}^{1}$ is the successor of a cardinal of cofinality $\omega$.

But so far, we do not know how to prove CL from Blackwell Determinacy.

\section{References}

[B169] D. Blackwell, Infinite $G_{\delta}$ games with imperfect information, Polska Akademia Nauk — Instytut Matematyczny — Zastosowania Matematyki, 10 (1969), 99-101, MR 0243837, Zbl 0232.90068.

[B197] _ Games with infinitely many moves and slightly imperfect information, in 'Games of No Chance' (R.J. Nowakowski, ed.), Combinatorial Games at MSRI, Workshop, July 11-21, 1994 in Berkeley, CA, USA, Cambridge, 1997, 407-408, Zbl 0873.90115.

[Ha78] L.A. Harrington, Analytic determinacy and $0^{\#}$, J. Symbolic Logic, 43 (1978), 685-693, MR 0518675, Zbl 0398.03039.

[Ka94] A. Kanamori, The Higher Infinite, Large Cardinals in Set Theory from Their Beginnings, Perspectives in Mathematical Logic, Berlin, 1994, MR 1321144, Zbl 0813.03034.

[Ke73] A.S. Kechris, Measure and category in effective descriptive set theory, Ann. Math. Logic, 5 (1973), 337-384, MR 0369072, Zbl 0277.02019.

[Ke78] _ AD and projective ordinals, in 'Cabal Seminar 76-77' (A.S. Kechris and Y.N. Moschovakis, eds.), Proceedings, Caltech-UCLA Logic Seminar 1976-77, Lecture Notes in Mathematics, 689, Berlin, 1978, 91-132, MR 0526915, Zbl 0434.03036.

[K177] E.M. Kleinberg, Infinitary Combinatorics and the Axiom of Determinateness, Lecture Notes in Mathematics, 612, Berlin, 1977, MR 0479903, Zbl 0362.02067.

[Lö02a] B. Löwe, Playing with mixed strategies on infinite sets, Internat. J. Game Theory, 31 (2002), 137-150, MR 1939050.

[Lö02b] _ Consequences of Blackwell determinacy, Irish Math. Soc. Bull., 49 (2002),43-69, MR 1960042.

[Ma98] D.A. Martin, The determinacy of Blackwell games, J. Symbolic Logic, 63 (1998), 1565-1581, MR 1665779, Zbl 0926.03071

[MaNeVe03] D.A. Martin, I. Neeman and M. Vervoort, The strength of Blackwell determinacy, J. Symbolic Logic, 68 (2003), 615-636, MR 1976594.

[Mo80] Y.N. Moschovakis, Descriptive Set Theory, Studies in Logic and the Foundations of Mathematics, 100, North-Holland, Amsterdam, 1980, MR 0561709, Zbl 0433.03025.

[StWe98] J.R. Steel and P.D. Welch, $\Sigma_{3}^{1}$ absoluteness and the second uniform indiscernible, Israel J. Math., 104 (1998), 157-190, MR 1622295, Zbl 0915.03042.

[Ve95] M.R. Vervoort, Blackwell Games, Doctoraalscriptie, Universiteit van Amsterdam, November 1995. 
[Ve96] _ Blackwell games, in 'Statistics, probability, and game theory: Papers in honor of David Blackwell' (T.S. Ferguson, L.S. Shapley and J.B. MacQueen, eds.) Hayward CA, Institute of Mathematical Statistics Lecture Notes-Monograph Series, 30, 369-390, MR 1481790.

[Ve00]_ Games, Walks and Grammars, Problems I've worked on, Academisch Proefschrift ter verkrijging van de graad van doctor aan de Universiteit van Amsterdam, 2000.

Received April 15, 2003 and revised August 8, 2003. The author would like to thank Tony Martin (Los Angeles CA) for his guidance. This work has been made possible by numerous travel grants awarded by the Studienstiftung des deutschen Volkes, the Association for Symbolic Logic, and the Graduiertenkolleg "Algebraische, analytische und geometrische Methoden und ihre Wechselwirkung in der modernen Mathematik" and the Deutsche Forschungsgemeinschaft (828/2001-LO834/2-1). Part of this work was done while the author was financed by a grant of the Studienstiftung des deutschen Volkes.

\section{INSTITUTE FOR LOGIC}

LANGUAGE AND COMPUTATION

UNIVERSITEIT VAN AMSTERDAM

Plantage Muidergracht 24

1018 TV AMSTERDAM

The Netherlands

E-mail address: bloewe@science.uva.nl 